\title{
Mass Flow Estimation with Model Bias Correction for a Turbocharged Diesel Engine
}

\author{
Tomáš Polóni*, Boris Rohal-Ilkiv \\ Institute of Automation, Measurement and Applied Informatics \\ Faculty of Mechanical Engineering, Slovak University of Technology \\ 812 31 Bratislava, Slovakia \\ Email: tomas.poloni@stuba.sk, boris.rohal-ilkiv@stuba.sk \\ Tor Arne Johansen \\ Center for Autonomous Marine Operations and Systems (AMOS) \\ Department of Engineering Cybernetics \\ Norwegian University of Science and Technology \\ N-7491 Trondheim, Norway \\ Email: tor.arne.johansen@itk.ntnu.no
}

\begin{abstract}
A systematic design method for mass flow estimation with correction for model bias is proposed. Based on an augmented observable Mean Value Engine Model (MVEM) of a turbocharged Diesel engine, the online estimation of states with additional biases is performed to compute the mass flows for different places. A correction method is applied, that utilizes estimated biases which are in a least-square sense redistributed between the correction terms to the uncertain mass flow maps and then added to the estimated mass flows. An Extended Kalman Filter (EKF) is tested off-line on production car engine data where the combination of an intake manifold pressure sensor, exhaust manifold pressure sensor and turbocharger speed sensor is compared and discussed in different sensor fusions. It is shown that the correction method improves the uncorrected estimated air mass flow which is validated against the airflow data measured in the intake duct.
\end{abstract}

Keywords: Diesel engine, Mass flow estimation, Bias estimation, Kalman filtering, Mean value engine model, Model correction method

${ }^{*}$ Corresponding author 


\section{Introduction}

Accurate information about the air charge of turbocharged Diesel engines is important for the fueling control where the injected amount of fuel can cause a visible smoke due to incomplete combustion, if the air charge quantity is overestimated. On the other side, the performance can suffer if the fueling is too conservative due to underestimating the air charge quantity. The mass charge induced by the cylinders is defined by the air quantity from the compressor flow and the exhaust gas recirculation (EGR) mass flow. The quality of the induced mass charge is defined by the air-fuel ratio. The information about the true amount of air charge is dimmed by premixing the EGR mass flow to the cylinders, containing the burned gas and unburned fresh air. The percentage of the fresh air in the EGR mass flow can be estimated via knowledge of burned gas fraction dynamics (Diop et al., 1999; Wang, 2008). The most desirable estimation technique combines the mass flow estimation (especially the EGR flow) and the oxygen concentration estimation to estimate the complete information about the air charge for a turbocharged Diesel engine (Kang et al., 2011) or for direct injection spark ignition engine (Stotsky and Kolmanovsky, 2002). These two problems of in-cylinder mass flow estimation and in-cylinder oxygen concentration estimation are often treated as independent estimation problems. The estimation of air charge for turbocharged Diesel engines with no EGR system with measured intake pressure (MAP) and intake temperature is reported in Storset et al. (2004). For the same kind of engine, the estimation of air charge through the adaptation of volumetric efficiency based on the upstream compressor airflow measurement (MAF) and MAP measurement is documented in Stefanopoulou et al. (2004). The compressor flow to the intake manifold can be estimated with MAP sensor and exhaust manifold pressure (EXMP) sensor (van Nieuwstadt et al., 2000; Kolmanovsky et al., 2000; Polóni et al., 2012), where van Nieuwstadt et al. (2000) base the EGR control loop on the estimated compressor flow information. The improved mass flow estimation (in-cylinder mass flow, compressor mass flow and EGR mass flow) accuracy is achieved with additional measurement of the turbocharger speed (NTC) (Höckerdal et al., 2009; Polóni et al., 2012). The MAF sensor adaptation based on the MAF measurement and turbocharger speed signal is studied in Höckerdal et al. (2011), where the measured air-mass flow with a production 
MAF sensor is imprecise and requires corrections. A promising approach to estimate the quantity of the induced charge is in application of the incylinder pressure measurement, however so far the experiments are reported under steady state conditions (Desantes et al., 2010).

The precision of the engine's mass flow estimation is characterized by the magnitude of the error. A model-based mass flow estimation algorithms relies on engine model precision which is sometimes uncertain due to mapbased steady state Mean-Value-Engine-Model (MVEM) identification principles (Jung, 2003; Eriksson et al., 2010) and engine aging. The adaptation scheme (Stefanopoulou et al., 2004), for engines without the EGR, considers the adaptation of the volumetric efficiency where adaptation scheme for a naturally aspired spark ignition engine with EGR in Kolmanovsky et al. (2006) suggests to adapt in-cylinder mass flow based on the MAF and MAP measurements. An approach founded on the linearized state-space model augmentation with variable structure of model bias terms is proposed in Höckerdal et al. (2009) where the augmentation with a given structure of model bias terms is proposed in Polóni et al. (2012). The augmentations (Höckerdal et al., 2009; Polóni et al., 2012) aim to improve the estimation of the intake manifold pressure, exhaust manifold pressure and turbocharger speed by additional estimation of the bias terms entering the nonlinear statespace model.

For the air mass flow estimation problem, different possibilities exist:

- the use of a MAF sensor

- the use of a MAF and MAP sensor with an observer/model,

- the use of an Extended Kalman Filter (EKF) and MVEM with fusion of different sensor sets (MAP, EXMP, NTC) (as presented in this paper)

- the use of an exhaust lambda sensor (EGO) and injection signal for estimating the air mass flow

- other combinations of the previous ones

This work presents novelty in utilization of the estimated biases for the mass flow computation and presents the correction method that is experimentally tested on the turbocharged Diesel engine data. As we have focused on the bias modeling and redistribution of the bias error to mass flow maps, the optimal choice of estimation algorithm is not emphasized in our research. 


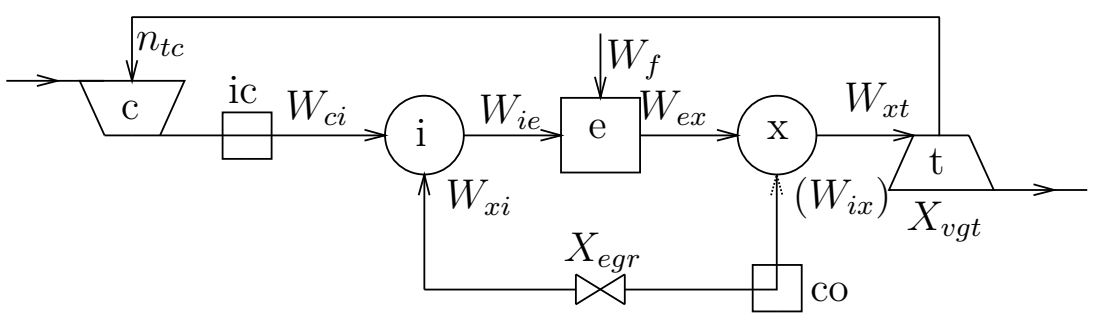

Figure 1: Schematic turbocharged Diesel engine model representation

The Extended Kalman Filter (EKF) is chosen for estimation since it is a widely established and well known engineering tool. The computation of the mass flow correction vector is formulated as a least-squares problem under the assumption that map-based mass flow computations are the most uncertain parts of the model. Further contribution of this paper is the methodology of using the EKF, MVEM and the sensor fusion for on-board estimation of (not only) upstream compressor airflow, allowing to omit the MAF sensor.

\section{Turbocharged engine system description and Mean-Value En- gine model}

The simplified schematic turbocharged Diesel engine setup considered in this study is shown in Figure 1. According to this figure, the engine is modeled with the compressor (c), turbine (t), final volume intake manifold (i), final volume exhaust manifold (x), intercooler (ic), EGR cooler (co), EGR pipe and the engine cylinders (e). The model has five states. The first four states represent the mass dynamics and they are: $p_{i}$ intake manifold pressure $[k P a], m_{i}$ intake mass $[k g], p_{x}$ exhaust manifold pressure $[k P a]$ and $m_{x}$ exhaust mass $[\mathrm{kg}]$. The fifth state is the turbocharger speed $n_{t c}[\mathrm{rpm}]$. The inputs are EGR valve position $X_{\text {egr }}$ [\% open], VGT actuator position $X_{v g t}\left[\%\right.$ open], injected fuel mass $W_{f}\left[\mathrm{~kg}_{\mathrm{s}} \mathrm{s}^{-1}\right]$ and the engine speed $n_{e}[\mathrm{rpm}]$.

The engine model is represented by the following mass and energy balance differential equations in the intake and exhaust manifolds, and by the torque 
balance at the turbocharger shaft (Kolmanovsky et al., 1998)

$$
\begin{aligned}
\dot{p}_{i} & =\frac{R \kappa}{V_{i}}\left(W_{c i} T_{c i}-W_{i e} T_{i}+W_{x i} T_{x i}-\frac{\dot{Q}_{1}}{c_{p}}\right) \\
\dot{m}_{i} & =W_{c i}-W_{i e}+W_{x i} \\
\dot{p}_{x} & =\frac{R \kappa}{V_{x}}\left(\left(W_{i e}+W_{f}\right) T_{e}-\left(W_{x i}+W_{x t}\right) T_{x}-\frac{\dot{Q}_{2}}{c_{p}}\right) \\
\dot{m}_{x} & =W_{i e}+W_{f}-W_{x i}-W_{x t} \\
\dot{n}_{t c} & =\left(\frac{60}{2 \pi}\right)^{2} \frac{1}{J}\left(\frac{P_{t}-P_{c}}{n_{t c}}\right)
\end{aligned}
$$

The symbols in these equations are either input variables, state variables, constants or functions of the five states and inputs. The index associated with each variable defines the location of the variable. In the case of two indexes the first one is the upstream and the second one is the downstream location. The mass flows are denoted as $W\left[\mathrm{~kg}_{\mathrm{s}} \mathrm{s}^{-1}\right]$. The EGR mass flow is modeled as $W_{x i}$ where alternatively the backflow can be considered as $W_{i x}$ however, under standard operating conditions $W_{i x}=0$. The temperatures $[K]$ in the intake and exhaust manifolds are $T_{i}$ and $T_{x}$. The differences of the static and dynamic pressures and temperatures are neglected because of the low mass flows. The constant parameters for the model are the intake manifold volume $V_{i}\left[\mathrm{~m}^{3}\right]$, exhaust manifold volume $V_{x}\left[\mathrm{~m}^{3}\right]$, specific heats at constant pressure and volume $\left[J . \mathrm{kg}^{-1} \cdot K^{-1}\right] c_{p}, c_{v}$, isentropic exponent of air $\kappa=\frac{c_{p}}{c_{v}}$, specific gas constant of air $R=c_{p}-c_{v}$ and the turbocharger inertia $J\left[\mathrm{~kg} \cdot \mathrm{m}^{2}\right]$. The heat losses in the Eq. (1), $\dot{Q}_{1}$ and Eq. (3), $\dot{Q}_{2}$ are assumed to be zero. For the intake manifold, this is a reasonable assumption since the temperature is not much different than the ambient temperature. However in the case of exhaust manifold the heat loses are significant. The exhaust gas heat loss is implicitly captured in the steady state (look-up table) out-flow engine temperature model $T_{e}$ that is discussed later.

The following equations summarize the dependencies of intermediate variables in Eq. (1)-(5). Some of these dependencies are expressed as the lookup tables obtained by fitting the steady-state experimental engine data to a second order polynomial surface with parameter vector $\Theta$. The others are obtained by physical relations. The air mass flow from compressor to the intake manifold $W_{c i}$, pressure after compressor $p_{c}$ and the compressor efficiency 
$\eta_{c}$ are mapped as (Jung, 2003)

$$
\begin{gathered}
W_{c i}=f_{W_{c i}}\left(p_{i}, n_{t c}, \Theta_{W_{c i}}\right) \\
p_{c}=f_{p_{c}}\left(W_{c i}, n_{t c}, \Theta_{p_{c}}\right) \\
\eta_{c}=f_{\eta_{c}}\left(W_{c i}, p_{i}, \Theta_{\eta_{c}}\right)
\end{gathered}
$$

with fitted polynomial surfaces characterizing the turbocharged Diesel combustion engine. The look-up tables are 2-D functions with a linear interpolation capability with grid data generated from the fitted polynomial surfaces.

The temperature after the compressor is given by

$$
T_{c}=T_{a}+\frac{1}{\eta_{c}} T_{a}\left[\left(\frac{p_{c}}{p_{a}}\right)^{\frac{\kappa-1}{\kappa}}-1\right]
$$

where $T_{a}$ is the ambient temperature and $p_{a}$ is the ambient pressure. The following mappings for the temperature after the intercooler $T_{c i}$ and the engine volumetric efficiency $\eta_{v}$ are

$$
\begin{gathered}
T_{c i}=f_{T_{c i}}\left(W_{c i}, T_{c}, \Theta_{T_{c i}}\right) \\
\eta_{v}=f_{\eta_{v}}\left(n_{e}, p_{i}, \Theta_{\eta_{v}}\right)
\end{gathered}
$$

with fitted polynomial surfaces. The mass flow from the intake manifold to the engine cylinders is

$$
W_{i e}=\frac{m_{i} n_{e} V_{d}}{V_{i} 120} \eta_{v}
$$

The temperatures in the intake and the exhaust manifolds are

$$
\begin{aligned}
T_{i} & =\frac{p_{i} V_{i}}{m_{i} R} \\
T_{x} & =\frac{p_{x} V_{x}}{m_{x} R}
\end{aligned}
$$

The EGR flow $W_{x i}$ is modeled by the orifice equation that is characterized by the effective flow area

$$
A_{e g r}=a_{2} X_{e g r}^{2}+a_{1} X_{e g r}
$$


where $a_{2}$ and $a_{1}$ are polynomial coefficients and by nonlinear term $\Psi_{\text {egr }}$ that reflects the pressure conditions upstream and downstream at the orifice (Heywood, 1988)

$$
\Psi_{e g r}=\left\{\begin{array}{c}
\sqrt{\frac{2 \kappa}{\kappa-1}\left[\left(\frac{p_{i}}{p_{x}}\right)^{\frac{2}{\kappa}}-\left(\frac{p_{i}}{p_{x}}\right)^{\frac{\kappa+1}{\kappa}}\right]} \text { if } \quad \frac{p_{i}}{p_{x}} \geq\left(\frac{2}{\kappa+1}\right)^{\frac{\kappa}{\kappa-1}} \\
\sqrt{\left(\kappa \frac{2}{\kappa+1}\right)^{\frac{\kappa+1}{\kappa-1}}} \text { otherwise } \\
W_{x i}=\frac{A_{e g r} p_{x}}{\sqrt{R T_{x}}} \Psi_{e g r}
\end{array}\right.
$$

The temperature of the mass that flows out of the cylinders $T_{e}$, EGR mass temperature $T_{x i}$ and the mass flow from the exhaust manifold to the turbine $W_{x t}$ are

$$
\begin{gathered}
T_{e}=f_{T_{e}}\left(W_{f}, W_{i e}, \Theta_{T_{e}}\right) \\
T_{x i}=f_{T_{x i}}\left(T_{x}, W_{x i}, \Theta_{T_{x i}}\right) \\
W_{x t}=f_{W_{x t}}\left(X_{v g t}, p_{x}, \Theta_{W_{x t}}\right)
\end{gathered}
$$

with fitted polynomial surfaces. The power of the compressor is given by

$$
P_{c}=W_{c i} c_{p} \frac{1}{\eta_{c}} T_{a}\left[\left(\frac{p_{c}}{p_{a}}\right)^{\frac{\kappa-1}{\kappa}}-1\right]
$$

The power of the turbine is calculated as

$$
P_{t}=W_{x t} c_{p} \frac{1}{\eta_{t}} T_{x}\left[1-\left(\frac{p_{t}}{p_{x}}\right)^{\frac{\kappa-1}{\kappa}}\right]
$$

where the turbine efficiency

$$
\eta_{t}=f_{\eta_{t}}\left(\frac{p_{t}}{p_{x}}, n_{t c}, \Theta_{\eta_{t}}\right)
$$

is mapped with the turbine pressure

$$
p_{t}=b_{3} W_{x t}^{3}+b_{2} W_{x t}^{2}+b_{1} W_{x t}+b_{0}
$$

where $b_{0}, \ldots, b_{3}$ are the polynomial coefficients. More details on model validation can be found in (Polóni et al., 2012). 


\section{Model bias augmentations}

The precision of the MVEM depends on its structure and parameters. The imprecision is given by the finite mean-value model structure that with limited accuracy gives a true picture of physical relations between different sub-systems of a turbocharged Diesel engine. The identification of the maps of the MVEM is experimentally performed at the engine steady state conditions. The identification in steady state conditions introduces certain systematic errors due to neglecting the dynamic effects of the modeled variables.

The open loop observer indicates with which state equations the modeling errors are associated with (Polóni et al., 2012). One way to remove these errors is to consistently inspect the parameter identification of the maps, to inspect the methods and conditions of the experiments (Wahlström and Eriksson, 2011) under which the maps were obtained and identify more accurate maps. This is a considerable task and does not account for structural model errors or errors due to aging of the engine. Another way to compensate for these modeling errors is to augment the model with bias parameters to be identified on-line by the observer or to introduce adaptive models (Höckerdal et al., 2011). Allocating bias terms to the state equations with the largest open loop modeling errors provides a first guess. In the experimental evaluation presented in section 5, careful validation considering alternative combination of bias term allocations showed that this provides good solutions. However, as each model and engine type may have very different modeling error characteristics, we recommend that careful validation with alternative combinations of bias terms is always performed. The number of such bias parameters is limited by the observability i. e. by the number of measurements available and by the structure of the equations (Höckerdal et al., 2009).

A compact form of an augmented state-space model is

$$
\begin{aligned}
\dot{x}_{s} & =\tilde{f}_{c}\left(x_{s}, w, u\right)+z_{x} \\
\dot{w} & =z_{w}
\end{aligned}
$$

where the continuous function $\tilde{f}_{c}$ has the mapping properties $\tilde{f}_{c}: \mathbb{R}^{n_{s}} \times \mathbb{R}^{n_{w}} \times$ $\mathbb{R}^{n_{u}} \rightarrow \mathbb{R}^{n_{s}}, n_{s}$ is the size of the state vector $x_{s}, n_{w}$ is the size of the bias vector $w$ and $n_{u}$ is the size of the input vector $u$. The state process noise vector is $z_{x}$ and the augmented state (bias) process noise vector is $z_{w}$. The 
augmented state vector $x \in \mathbb{R}^{n_{s}+n_{w}}$ is defined by

$$
x=\left[\begin{array}{l}
x_{s} \\
w
\end{array}\right]
$$

The state and bias equations can be combined as

$$
\dot{x}=f_{c}(x, u)+z
$$

where the continuous function $f_{c}$ has the mapping properties $f_{c}: \mathbb{R}^{n_{x}} \times$ $\mathbb{R}^{n_{u}} \rightarrow \mathbb{R}^{n_{x}}, n_{x}$ being the dimension of the augmented state vector $x$ and $z=\left[z_{x}, z_{w}\right]^{T}$. The observation equation may be written as

$$
y=h_{c}(x, u)+v
$$

where $y \in \mathbb{R}^{n_{y}}$ is the vector of measurements, $h_{c}: \mathbb{R}^{n_{x}} \times \mathbb{R}^{n_{u}} \rightarrow \mathbb{R}^{n_{y}}$ is the continuous measurement function and $n_{y}$ is the number of measured states. The measurement errors are modeled with the noise term $v \in \mathbb{R}^{n_{y}}$. The most frequent situation encountered in practice is when the system is governed by continuous-time dynamics and the measurements are obtained at discrete time instances. For the estimator formulation we consider the numerically discretized dynamic nonlinear system described by the equations

$$
\begin{aligned}
x_{t+1} & =f\left(x_{t}, u_{t}\right)+z_{t} \\
y_{t} & =h\left(x_{t}, u_{t}\right)+v_{t}
\end{aligned}
$$

for $t=0,1, \ldots$, where $x_{t} \in \mathbb{R}^{n_{x}}$ is the state vector, $u_{t} \in \mathbb{R}^{n_{u}}$ is the input vector and $z_{t} \in \mathbb{R}^{n_{z}}$ is the process noise vector. The numerical discretization algorithm is discussed in the Appendix A. The state vector is observed through the measurement equation (31) where $y_{t} \in \mathbb{R}^{n_{y}}$ is the observation vector and $v_{t} \in \mathbb{R}^{n_{y}}$ is a measurement noise vector.

In the following presentation, the additive bias terms have been chosen as a lumped representation of modeling errors since it allows straightforward use of the EKF for estimation, and straightforward distribution of the modeling error to the modeled mass flows. We do not claim that additive bias terms will in general lead to more accurate models than e.g. multiplicative parameters, but this choice makes the approach simple and the results shows that it is an effective choice allowing model accuracy to be improved in the case study. 


\subsection{Two-sensor setup with sensors for $p_{i}$ and $p_{x}$}

Two measured variables will be processed by the two-sensor setup observer which means that, at most, two biases can be added. Instead of a mathematical analysis of observability we note that the system is open loop asymptotically stable and the estimates would converge if there were no model errors. Two measurements give two additional equations that allow two unknowns (two bias variables) to be introduced and solved. A possible augmentation of the model where $x_{s}=\left[p_{i}, m_{i}, p_{x}, m_{x}, n_{t c}\right]^{T}=\left[x_{1}, x_{2}, x_{3}, x_{4}, x_{5}\right]^{T}$ is with bias placed on the intake manifold pressure and bias placed on the exhaust manifold pressure (Alt. 1).

Alt. 1: Biases $w_{i}$ and $w_{x}$. The augmented state-space model with the biases $w_{i}$ and $w_{x}$ is

$$
\begin{aligned}
\dot{x}_{1} & =\frac{R \kappa}{V_{i}}\left(W_{c i} T_{c i}-W_{i e} T_{i}+W_{x i} T_{x i}\right)+w_{i}+z_{1} \\
\dot{x}_{2} & =W_{c i}-W_{i e}+W_{x i}+z_{2} \\
\dot{x}_{3} & =\frac{R \kappa}{V_{x}}\left[\left(W_{i e}+W_{f}\right) T_{e}-\left(W_{x i}+W_{x t}\right) T_{x}\right]+w_{x}+z_{3} \\
\dot{x}_{4} & =W_{i e}+W_{f}-W_{x i}-W_{x t}+z_{4} \\
\dot{x}_{5} & =\left(\frac{60}{2 \pi}\right)^{2} \frac{1}{J}\left(\frac{P_{t}-P_{c}}{n_{t c}}\right)+z_{5} \\
\dot{w}_{i} & =z_{6} \\
\dot{w}_{x} & =z_{7}
\end{aligned}
$$

The open loop observer simulation shows that the model is most imprecise on the intake manifold pressure state and turbocharger speed state (Polóni

et al., 2012). The augmentation with bias terms placed on derivatives of these states is therefore logical with the two-sensor setup (Alt. 2). 
Alt. 2: Biases $w_{i}$ and $w_{t c}$. The augmented state-space model with the biases $w_{i}$ and $w_{t c}$ is

$$
\begin{aligned}
\dot{x}_{1} & =\frac{R \kappa}{V_{i}}\left(W_{c i} T_{c i}-W_{i e} T_{i}+W_{x i} T_{x i}\right)+w_{i}+z_{1} \\
\dot{x}_{2} & =W_{c i}-W_{i e}+W_{x i}+z_{2} \\
\dot{x}_{3} & =\frac{R \kappa}{V_{x}}\left[\left(W_{i e}+W_{f}\right) T_{e}-\left(W_{x i}+W_{x t}\right) T_{x}\right]+z_{3} \\
\dot{x}_{4} & =W_{i e}+W_{f}-W_{x i}-W_{x t}+z_{4} \\
\dot{x}_{5} & =\left(\frac{60}{2 \pi}\right)^{2} \frac{1}{J}\left(\frac{P_{t}-P_{c}}{n_{t c}}\right)+w_{t c}+z_{5} \\
\dot{w}_{i} & =z_{6} \\
\dot{w}_{t c} & =z_{7}
\end{aligned}
$$

\subsection{Three-sensor setup with sensors for $p_{i}, p_{x}$ and $n_{t c}$}

The third measured variable, the turbocharger speed, is additionally introduced to study its impact on overall estimation precision with the bias terms placed on the intake manifold pressure, exhaust manifold pressure and turbocharger speed (Alt. 3).

Alt. 3: Biases $w_{i}, w_{x}$ and $w_{t c}$. The augmented state-space model with the biases $w_{i}, w_{x}$ and $w_{t c}$ is

$$
\begin{aligned}
\dot{x}_{1} & =\frac{R \kappa}{V_{i}}\left(W_{c i} T_{c i}-W_{i e} T_{i}+W_{x i} T_{x i}\right)+w_{i}+z_{1} \\
\dot{x}_{2} & =W_{c i}-W_{i e}+W_{x i}+z_{2} \\
\dot{x}_{3} & =\frac{R \kappa}{V_{x}}\left[\left(W_{i e}+W_{f}\right) T_{e}-\left(W_{x i}+W_{x t}\right) T_{x}\right]+w_{x}+z_{3} \\
\dot{x}_{4} & =W_{i e}+W_{f}-W_{x i}-W_{x t}+z_{4} \\
\dot{x}_{5} & =\left(\frac{60}{2 \pi}\right)^{2} \frac{1}{J}\left(\frac{P_{t}-P_{c}}{n_{t c}}\right)+w_{t c}+z_{5} \\
\dot{w}_{i} & =z_{6} \\
\dot{w}_{x} & =z_{7} \\
\dot{w}_{t c} & =z_{8}
\end{aligned}
$$

\section{Mass flow correction method}

The basic strategy to correct the mass flows is shown in Figure 2. The jointly estimated state vector $x_{s}$ and bias vector $w$ is used to compute the 


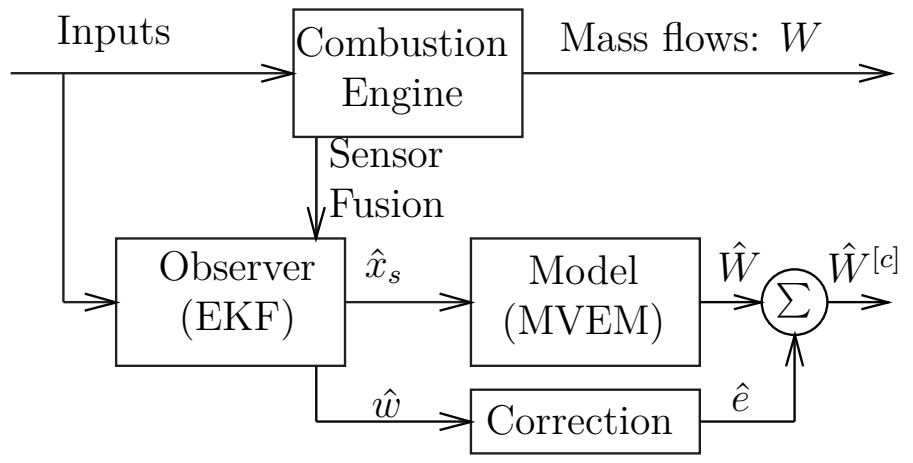

Figure 2: Mass flow estimation scheme with error-bias correction

mass flow vector and the correction vector respectively. The estimated mass flow vector $\hat{W}$ is corrected by the correction vector $\hat{e}$ to be explained in this section. The corrected mass flow vector is denoted $\hat{W}^{[c]}$.

The estimated bias vector $w$ represents the information about errors of the state equations. This error is called a model bias, although it is not a constant value but modeled by a Wiener process. This bias information can be redistributed in a least-square sense among the modeled mass flow terms $W_{c i}, W_{i e}, W_{x i}, W_{x t}$. The basis for the least-squares problem is that the mass flow maps $W_{c i}, W_{i e}, W_{x i}$ and $W_{x t}$ contains the most significant contributions to model error, as other sources are neglected in the redistribution.

The least-square error equations are formed by the subtraction of each state equation from the corrected state equation. For example, the subtraction of the following two equations derived from Eq. (32) leads to the first least-square error equation given by the Eq. (56)

$$
\begin{aligned}
& \frac{V_{i}}{R \kappa} \dot{x}_{1}=W_{c i} T_{c i}-W_{i e} T_{i}+W_{x i} T_{x i}+\frac{V_{i}}{R \kappa} w_{i} \\
& \frac{V_{i}}{R \kappa} \dot{x}_{1}=\left(W_{c i}+e_{c i}\right) T_{c i}-\left(W_{i e}+e_{i e}\right) T_{i}+\left(W_{x i}+e_{x i}\right) T_{x i}
\end{aligned}
$$

The least-square error equations are formed below each state equation as 
follows

$$
\begin{aligned}
& \frac{V_{i}}{R \kappa} \dot{x}_{1}=W_{c i} T_{c i}-W_{i e} T_{i}+W_{x i} T_{x i}+\frac{V_{i}}{R \kappa} w_{i} \\
& 0=e_{c i} T_{c i}-e_{i e} T_{i}+e_{x i} T_{x i}-\frac{V_{i}}{R \kappa} w_{i} \\
& \dot{x}_{2}=W_{c i}-W_{i e}+W_{x i}+\frac{V_{i}}{T_{S i} R \kappa} w_{i} \\
& 0=e_{c i}-e_{i e}+e_{x i}-\frac{V_{i}}{T_{S i} R \kappa} w_{i} \\
& \frac{V_{x}}{R \kappa} \dot{x}_{3}=\left(W_{i e}+W_{f}\right) T_{e}-\left(W_{x i}+W_{x t}\right) T_{x}+\frac{V_{x}}{R \kappa} w_{x} \\
& 0=e_{i e} T_{e}-e_{x i} T_{x}-e_{x t} T_{x}-\frac{V_{x}}{R \kappa} w_{x} \\
& \dot{x}_{4}=W_{i e}+W_{f}-W_{x i}-W_{x t}+\frac{V_{x}}{T_{S x} R \kappa} w_{x} \\
& 0=e_{i e}-e_{x i}-e_{x t}-\frac{V_{x}}{T_{S x} R \kappa} w_{x} \\
& \frac{J}{\left(\frac{60}{2 \pi}\right)^{2}} \dot{x}_{5}=W_{x t} \frac{c_{p} T_{x}}{n_{t c} \eta_{t}}\left[1-\left(\frac{p_{t}}{p_{x}}\right)^{\frac{\kappa-1}{\kappa}}\right]- \\
& -W_{c i} \frac{c_{p} T_{a}}{n_{t c} \eta_{c}}\left[\left(\frac{p_{c}}{p_{a}}\right)^{\frac{\kappa-1}{\kappa}}-1\right]+\frac{J}{\left(\frac{60}{2 \pi}\right)^{2}} w_{t c} \\
& 0=e_{x t} \frac{c_{p} T_{x}}{n_{t c} \eta_{t}}\left[1-\left(\frac{p_{t}}{p_{x}}\right)^{\frac{\kappa-1}{\kappa}}\right]- \\
& -e_{c i} \frac{c_{p} T_{a}}{n_{t c} \eta_{c}}\left[\left(\frac{p_{c}}{p_{a}}\right)^{\frac{\kappa-1}{\kappa}}-1\right]-\frac{J}{\left(\frac{60}{2 \pi}\right)^{2}} w_{t c}
\end{aligned}
$$

where the errors $e_{c i}, e_{i e}, e_{x i}, e_{x t}$ are elements of the correction vector $e$.

The physical relation of the pressure state equations and mass state equations is in the scaling of the flow terms $W$ by the temperatures $T$ and the $\frac{R \kappa}{V}$ term. If we consider the scaling temperature for the bias $w_{i}$ as $T_{S i}=\left(T_{c i}+T_{i}+T_{x i}\right) / 3$ and the scaling temperature for the bias $w_{x}$ as $T_{S x}=\left(T_{e}+T_{x}\right) / 2$, one can introduce the same bias term $(w)$ for the mass balance state equation. 
The linear least-square problem to estimate the correction vector is formulated as the Euclidean norm minimization problem

$$
\hat{e}=\min _{e}\|A e+b\|_{2}^{2}
$$

for the previously considered sensor and bias alternatives:

Two-sensor setup $\left(p_{i}, p_{x}\right)$ with biases $w_{i}$ and $w_{x}$ (Alt. 1). In this sensor configuration, where pressures $p_{i}, p_{x}$ are measured, two biases are placed on the differential equations of the intake manifold pressure $w_{i}$ and differential equation of the exhaust manifold pressure $w_{x}$.

$$
A=\left[\begin{array}{cccc}
T_{c i} & -T_{i} & T_{x i} & 0 \\
T_{S i} & -T_{S i} & T_{S i} & 0 \\
0 & T_{e} & -T_{x} & T_{x} \\
0 & T_{S x} & -T_{S x} & -T_{S x}
\end{array}\right] ; e=\left[\begin{array}{c}
e_{c i} \\
e_{i e} \\
e_{x i} \\
e_{x t}
\end{array}\right] ; b=\left[\begin{array}{c}
-\frac{V_{i}}{R \kappa} w_{i} \\
-\frac{V_{i}}{R \kappa} w_{i} \\
-\frac{V_{x}}{R \kappa} w_{x} \\
-\frac{V_{x}}{R \kappa} w_{x}
\end{array}\right]
$$

Two-sensor setup $\left(p_{i}, p_{x}\right)$ with biases $w_{i}$ and $w_{t c}$ (Alt. 2). In this sensor configuration where pressures $p_{i}, p_{x}$ are measured, two biases are placed on the differential equations of the intake manifold pressure $w_{i}$ and differential equation of the turbocharger speed $w_{t c}$. With the bias terms $w_{i}$ and $w_{t c}$, the correction vector contains only three elements, since $w_{t c}$ can not be scaled for any other equation.

$$
\begin{aligned}
A & =\left[\begin{array}{ccc}
T_{c i} & -T_{i} & 0 \\
T_{S i} & -T_{S i} & 0 \\
-\frac{c_{p} T_{a}}{n_{t c} \eta_{c}}\left[\left(\frac{p_{c}}{p_{a}}\right)^{\frac{\kappa-1}{\kappa}}-1\right] & 0 & \frac{c_{p} T_{x}}{n_{t c} \eta_{t}}\left[1-\left(\frac{p_{t}}{p_{x}}\right)^{\frac{\kappa-1}{\kappa}}\right]
\end{array}\right] ; e=\left[\begin{array}{c}
e_{c i} \\
e_{i e} \\
e_{x t}
\end{array}\right] ; \\
b & =\left[\begin{array}{c}
-\frac{V_{i}}{R \kappa} w_{i} \\
-\frac{V_{i}}{R \kappa} w_{i} \\
-\frac{J}{\left(\frac{60}{2 \pi}\right)^{2}} w_{t c}
\end{array}\right]
\end{aligned}
$$

Three-sensor setup $\left(p_{i}, p_{x}, n_{t c}\right)$ with biases $w_{i}, w_{x}$ and $w_{t c}$ (Alt. 3). In this sensor configuration, pressures $p_{i}, p_{x}$ and the turbocharger speed $n_{t c}$ are measured, where the biases are $w_{i}, w_{x}$ and $w_{t c}$.

$$
A=\left[\begin{array}{cccc}
T_{c i} & -T_{i} & T_{x i} & 0 \\
T_{S i} & -T_{S i} & T_{S i} & 0 \\
0 & T_{e} & -T_{x} & T_{x} \\
0 & T_{S x} & -T_{S x} & -T_{S x} \\
-\frac{c_{p} T_{a}}{n_{t c} \eta_{c}}\left[\left(\frac{p_{c}}{p_{a}}\right)^{\frac{\kappa-1}{\kappa}}-1\right] & 0 & 0 & \frac{c_{p} T_{x}}{n_{t c} \eta_{t}}\left[1-\left(\frac{p_{t}}{p_{x}}\right)^{\frac{\kappa-1}{\kappa}}\right]
\end{array}\right]
$$




$$
e=\left[\begin{array}{c}
e_{c i} \\
e_{i e} \\
e_{x i} \\
e_{x t}
\end{array}\right] ; b=\left[\begin{array}{c}
-\frac{V_{i}}{R \kappa} w_{i} \\
-\frac{V_{i}}{R \kappa} w_{i} \\
-\frac{V_{x}}{R \kappa} w_{x} \\
-\frac{V_{x}}{R \kappa} w_{x} \\
-\frac{J^{2}}{\left(\frac{60}{2 \pi}\right)^{2}} w_{t c}
\end{array}\right]
$$

The elements of the $A$-matrix are obtained by applying the state vector estimate (given by the observer $\hat{x}_{s}$ ) to the MVEM; for example the intake manifold temperature given by the Eq. (13) is $T_{i}=\hat{p}_{i} V_{i} / \hat{m}_{i} R$.

The least-square problem is solved by the ordinary least-square closedform expression

$$
\hat{e}=\left(A^{T} A\right)^{+} A^{T} b
$$

Since the problem might be ill-conditioned the pseudoinverse of the matrix $A^{T} A$ is computed via the Moore-Penrose pseudoinverse through the singular value decomposition (SVD) (Golub and Van Loan, 1996) as

$$
\left(A^{T} A\right)^{+}=V D^{+} U^{T}
$$

where $V$ and $U$ are unitary matrices and $D$ is a diagonal matrix with nonnegative real numbers on the diagonal, the singular values, where the tolerance on singular values is $\delta_{S V D}$. Any singular value less than this tolerance, has its inverse set to zero. The $A$-matrix can be considered as a sensitivity-matrix where further sensitivity study can be performed to inspect the bias placement or inspect the parameter adaptation (if adaptive model is considered).

The mass flow computations are based on the state vector estimate computed by the EKF. After obtaining the estimated state vector from the EKF $\hat{x}_{s}=\left[\hat{p}_{i}, \hat{m}_{i}, \hat{p}_{x}, \hat{m}_{x}, \hat{n}_{t c}\right]^{T}$, and computing the correction vector $\hat{e}$ (Eq. 62), the corrected mass flow vector $\hat{W}^{[c]}$ is computed as follows

$$
\begin{gathered}
\hat{W}_{c i}^{[c]}=f_{W_{c i}}\left(\hat{p}_{i}, \hat{n}_{t c}, \Theta_{W_{c i}}\right)+\hat{e}_{c i} \\
\hat{W}_{i e}^{[c]}=\frac{\hat{m}_{i} n_{e} V_{d}}{V_{i} 120} \eta_{v}+\hat{e}_{i e} \\
\hat{W}_{x i}^{[c]}=\frac{A_{e g r} \hat{p}_{x}}{\sqrt{R \hat{T}_{x}}} \hat{\Psi}_{e g r}+\hat{e}_{x i} \\
\hat{W}_{x t}^{[c]}=f_{W_{x t}}\left(X_{v g t}, \hat{p}_{x}, \Theta_{W_{x t}}\right)+\hat{e}_{x t}
\end{gathered}
$$




\begin{tabular}{ccc} 
& RSE of $\hat{W}_{c i}$ & RSE of $\hat{W}_{c i}^{[c]}$ \\
\hline Alt. 1 & 0.147 & 0.139 \\
Alt. 2 & 0.112 & 0.096 \\
Alt. 3 & 0.093 & 0.067 \\
\hline
\end{tabular}

Table 1: Root Square Error (RSE) of uncorrected $\hat{W}_{c i}$ and corrected $\hat{W}_{c i}^{[c]}$ compressor air-flow estimate for different sensor/bias alternatives

\section{Experimental evaluation}

The presented mass flow estimation and correction strategy is tested offline on driving cycle engine data introduced in the Appendix B.1.

The quality of the algorithm is evaluated by the Root Square Error (RSE)

$$
R S E=\sqrt{\sum_{t=1}^{n}\left(M A F_{t}-\hat{W}_{c i, t}\right)^{2}}
$$

where $n=800$. The RSE is computed for the compressor air-flow $W_{c i}$, for which the validation measurement is available from the MAF sensor. The engine's other mass flows can not be easily measured, therefore it is assumed here that if the compressor flow is relatively precise the other mass flows are likely to be precise as well. The RSE index is computed for three considered sensor/bias alternatives, as shown in Table 1. It can be seen in Table 1, that in all three studied cases the correction method improves the estimated air-flows.

Two-sensor setup $\left(p_{i}, p_{x}\right)$ with biases $w_{i}$ and $w_{x}$ (Alt. 1). The estimated biases with the two-sensor setup of the intake and exhaust pressures are shown in Figure 3. The estimated compressor mass flow without the correction $\hat{W}_{c i}$ and the estimated compressor mass flow with the correction $\hat{W}_{c i}^{[c]}$ are compared in Figure 4. In this case, the uncorrected air-flow estimate $\hat{W}_{c i}$ is not very precise mostly during the downspeeding of the engine. Also the placement of biases does not provide sufficient error information toward the true compressor air flow which leads to biased corrected air-flow estimate $\hat{W}_{c i}^{[c]}$ as well.

Two-sensor setup $\left(p_{i}, p_{x}\right)$ with biases $w_{i}$ and $w_{t c}$ (Alt. 2). In this alternative, improvement of the estimated compressor air-flow is achieved. The estimated biases with the two-sensor setup of the intake and exhaust 


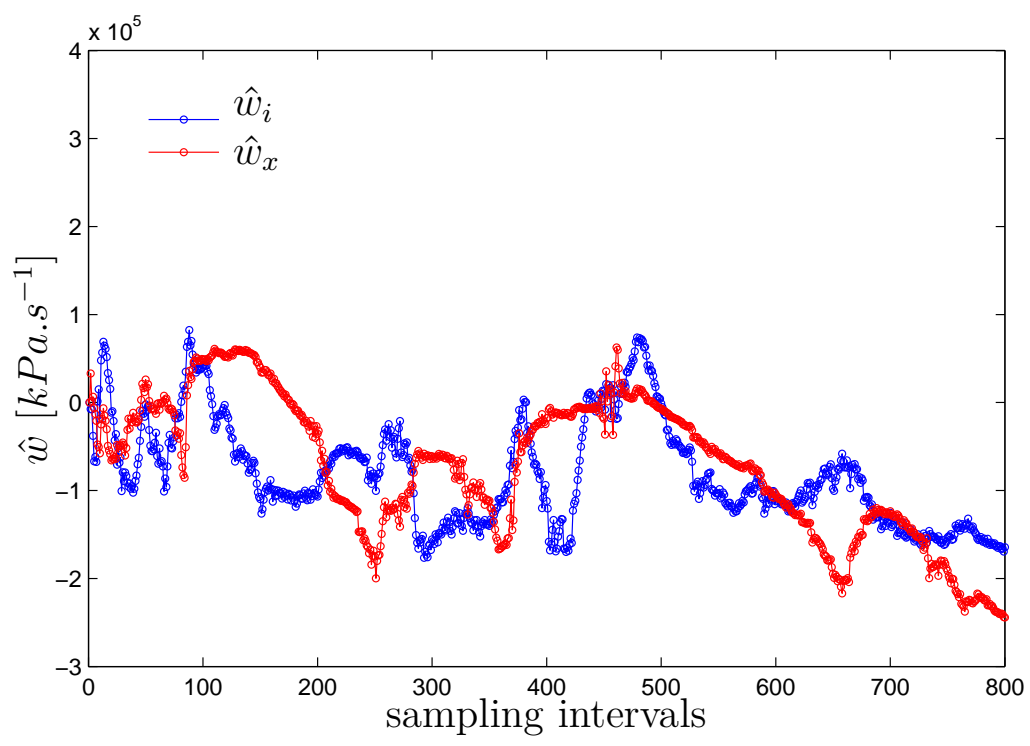

Figure 3: Alt. 1: Estimated biases with two-sensor measurement $\left(p_{i}, p_{x}\right)$

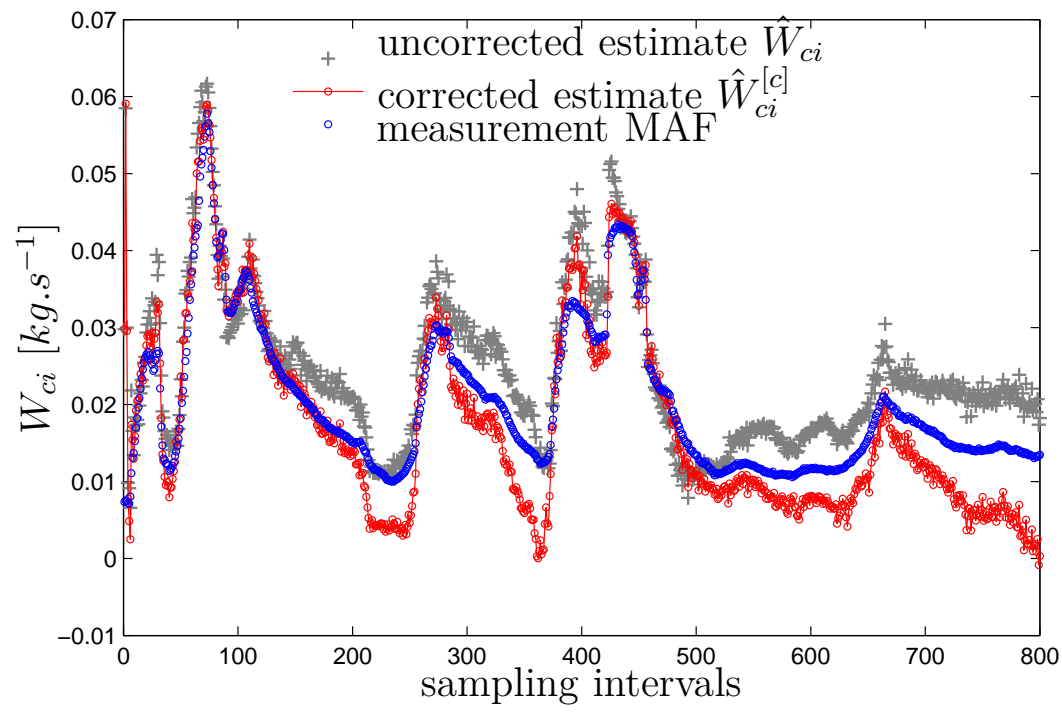

Figure 4: Alt. 1: Evaluation measurement and estimated compressor flow with two-sensor measurement $\left(p_{i}, p_{x}\right)$ 


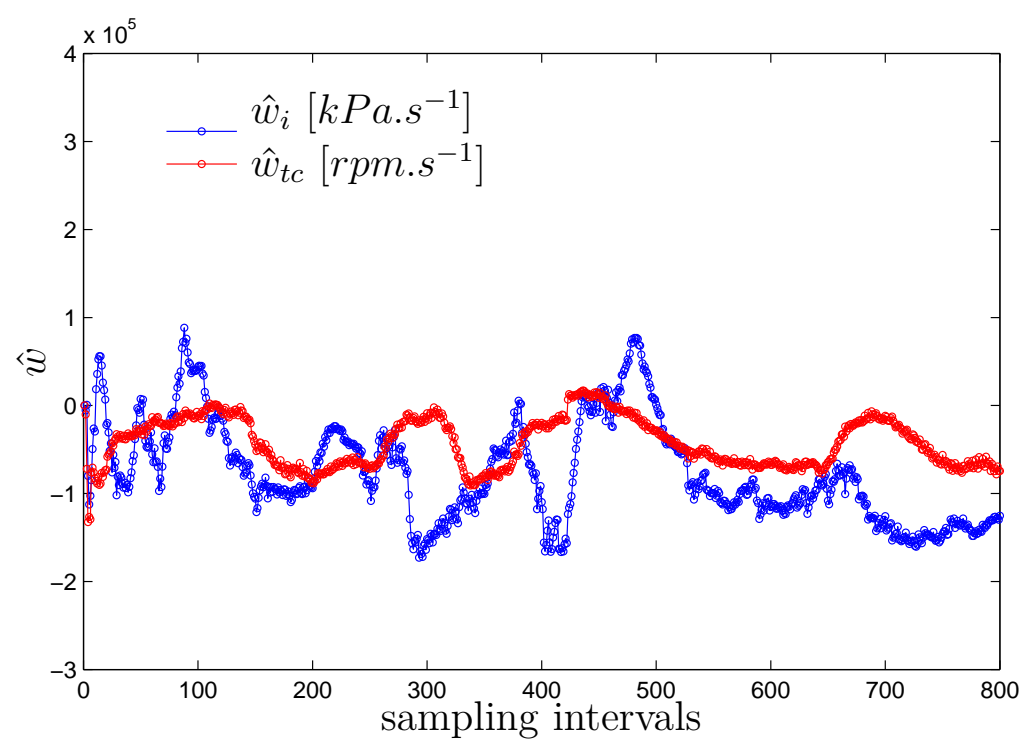

Figure 5: Alt. 2: Estimated biases with two-sensor measurement $\left(p_{i}, p_{x}\right)$

pressures are shown in Figure 5. The estimated compressor mass flow without the correction $\hat{W}_{c i}$ and the estimated compressor mass flow with the correction $\hat{W}_{c i}^{[c]}$ are compared in Figure 6. The improved estimates are due to the bias $w_{t c}$ that compensates better for the error of the turbocharger speed differential equation.

Three-sensor setup $\left(p_{i}, p_{x}, n_{t c}\right)$ with biases $w_{i}, w_{x}$ and $w_{t c}$ (Alt. 3). The best performance is obtained for the three-sensor setup alternative. This is expected, since the additional turbocharger speed sensor and additional bias, compared to Alt. 1 and Alt 2., provides additional information which is processed by the observer and the correction algorithm. The estimated biases are shown in Figure 7. The estimated compressor mass flow without the correction $\hat{W}_{c i}$ and the estimated compressor mass flow with the correction $\hat{W}_{c i}^{[c]}$ are compared in Figure 8.

\section{Conclusion}

A correction method for mass flow quantities of a turbocharged combustion engine is developed. Different combination of the production sensors 


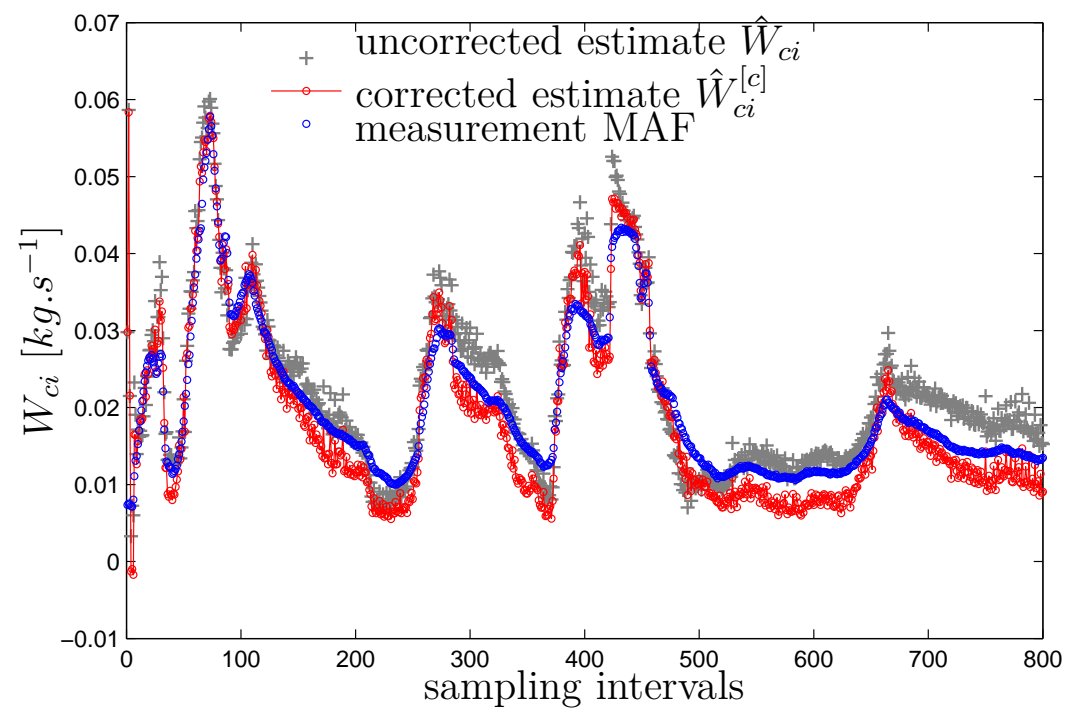

Figure 6: Alt. 2: Evaluation measurement and estimated compressor flow with two-sensor measurement $\left(p_{i}, p_{x}\right)$

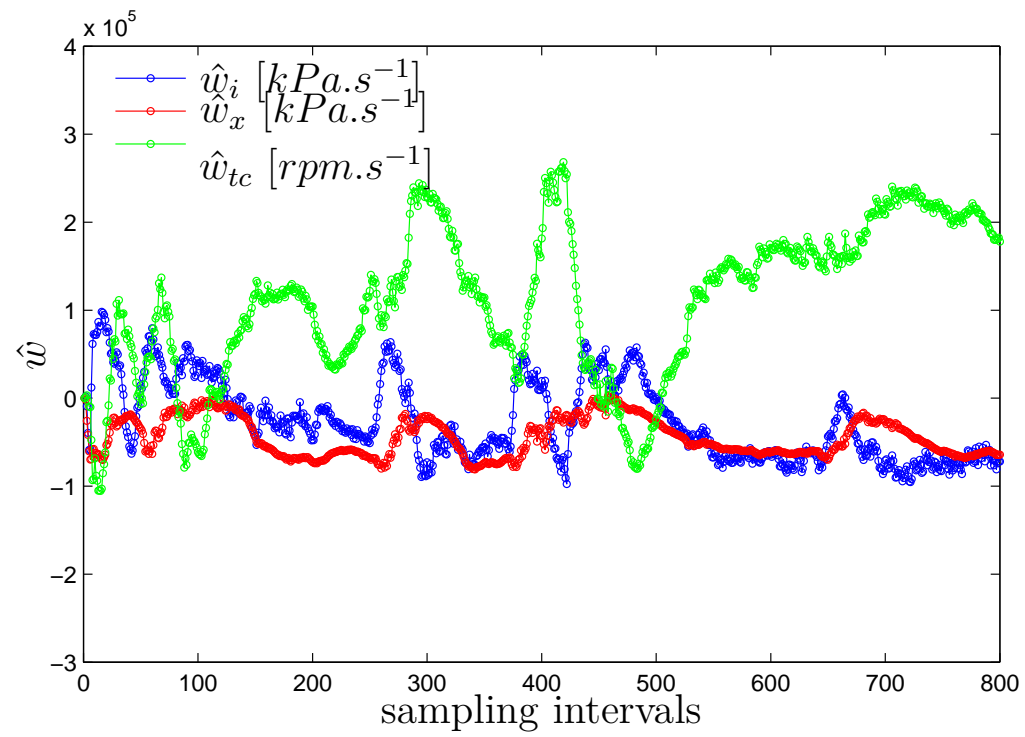

Figure 7: Alt. 3: Estimated biases with three-sensor measurement $\left(p_{i}, p_{x}, n_{t c}\right)$ 


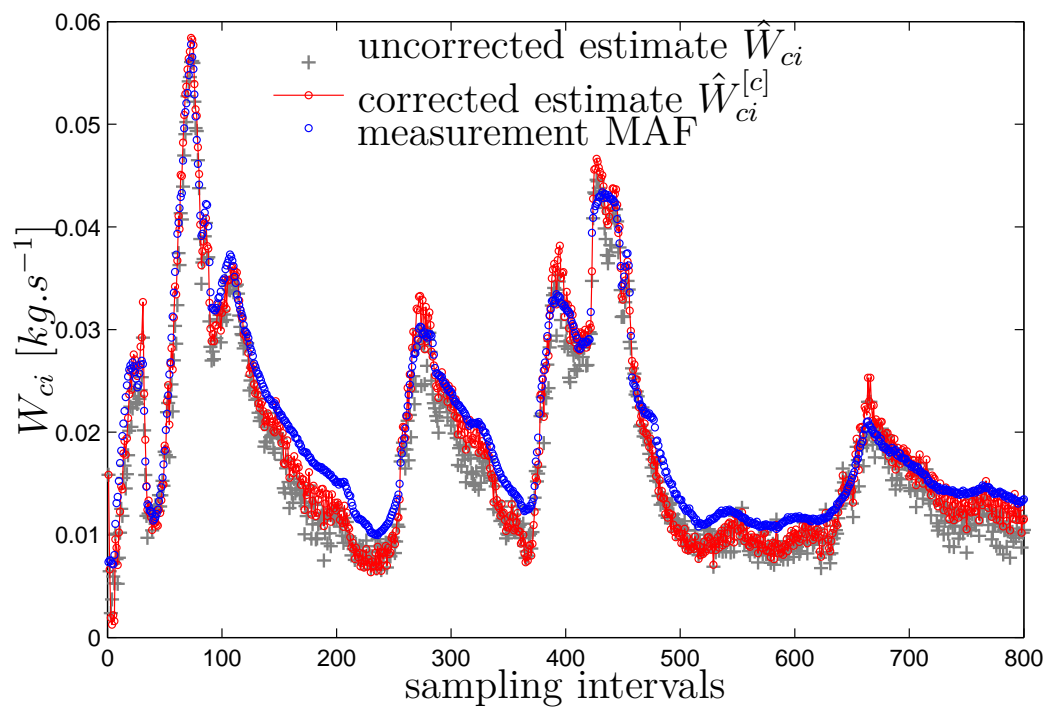

Figure 8: Alt. 3: Evaluation measurement and estimated compressor flow with threesensor measurement $\left(p_{i}, p_{x}, n_{t c}\right)$

(MAP, EXMP and NTC) can form the measurement vector, which is directly processed by the EKF to compute the augmented state vector with model states and model biases. The mass flows, computed based on the EKF observed states, are further corrected based on the EKF observed biases. In the off-line numerical experiment it is demonstrated, with the production car turbocharged Diesel engine data, that the mass flow correction method significantly improves the estimated air mass flow $W_{c i}$. The other mass flows: the EGR mass flow $W_{x i}$, the mass flow from the intake manifold to the engine cylinders $W_{i e}$ and the mass flow from the exhaust manifold to the turbine $W_{x t}$ are estimated as well, however they are not validated in this paper and therefore not discussed. It has been shown that the two-sensor setups with the measured intake manifold pressure and exhaust manifold pressure provide engine's mass flows estimate which precision can be further improved by the additional turbocharger speed measurement in the three-sensor setup. The proposed sensor fusion does not rely on the imprecise (or expensive) production car airflow measurement (MAF). In order to manage effects such as engine ageing, an implementation of the method in a production engine should be accompanied with an online adaptation of slowly varying model parameters. In this way parametric errors do not degrade the mass flow 
estimates, since they will not strongly influence the bias estimates.

Acknowledgment. T. P. acknowledges the support for this project from Prof. Luigi del Re, Institute for Design and Control of Mechatronical Systems, Johannes Kepler University, Linz. This work is supported by the Slovak Research and Development Agency under projects APVV-0090-10 and LPP0118-09. This work is also supported by the Norwegian Research Council and the Norwegian University of Science and Technology.

\section{Appendix A. Extended Kalman Filter}

The EKF is applied for estimation of the augmented state of the turbocharged Diesel engine. The following algorithm is in the literature known as the continuous-discrete or hybrid EKF (Brown and Hwang, 2012; Gelb et al., 2001). The dynamic system is given by Eq. (30) and Eq. (31). The process and the measurement noise is

$$
z_{t} \sim N\left(0, Q_{t}\right) \quad v_{t} \sim N\left(0, R_{t}\right)
$$

where $Q_{t}$ is the process noise covariance matrix and $R_{t}$ is the measurement noise covariance matrix. The initial condition of the state vector is $x_{0} \sim$ $N\left(\hat{x}_{0}^{+}, P_{0}^{+}\right)$. The estimate of the state vector at $t=0$ begins with the initial state vector estimate and with the initial covariance matrix of the initial state vector estimate error

$$
\begin{aligned}
\hat{x}_{0}^{+} & =E\left[x_{0}\right] \\
P_{0}^{+} & =E\left[\left(x_{0}-\hat{x}_{0}^{+}\right)\left(x_{0}-\hat{x}_{0}^{+}\right)^{T}\right]
\end{aligned}
$$

From time instance $t-1$, the dynamic system is simulatively propagated one step ahead as

$$
\hat{x}_{t}^{-}=f\left(\hat{x}_{t-1}^{+}, u_{t-1}\right)
$$

where $t=1,2, \ldots$. This one step computation is an a priori state estimate. The time update of the covariance matrix estimate is given by

$$
\dot{P}=Z(\hat{x}) P+P Z^{T}(\hat{x})+Q
$$

where

$$
Z(\hat{x})=\left.\frac{\partial f_{c}(x)}{\partial x}\right|_{x=\hat{x}}
$$


and $Q$ is a spectral density matrix, where $Q=\frac{1}{T} Q_{t}$ and $T$ is the sampling interval. The covariance matrix estimate of the state vector $\hat{x}_{t}^{-}$estimation error is achieved by simulative propagation of Eq. (A.5)

$$
P_{t}^{-}=g\left(P_{t-1}^{+}, Z\left(\hat{x}_{t-1}^{+}\right)\right)
$$

The EKF gain matrix of time instant $t$ is given by

$$
K_{t}=P_{t}^{-} L_{t}^{T}\left[L_{t} P_{t}^{-} L_{t}^{T}+M_{t} R_{t} M_{t}^{T}\right]^{-1}
$$

and the obtained measurement $y_{t}$ is used for state vector estimation (a posteriori estimate)

$$
\hat{x}_{t}^{+}=\hat{x}_{t}^{-}+K_{t}\left[y_{t}-h\left(\hat{x}_{t}^{-}\right)\right]
$$

The covariance matrix of the estimation error of $\hat{x}_{t}^{+}$is computed as

$$
P_{t}^{+}=\left[I-K_{t} L_{t}\right] P_{t}^{-}\left[I-K_{t} L_{t}\right]^{T}+K_{t} M_{t} R_{t} M_{t}^{T} K_{t}^{T}
$$

where

$$
L_{t}\left(\hat{x}_{t}^{-}\right)=\left.\frac{\partial h\left(x_{t}\right)}{\partial x_{t}}\right|_{x_{t}=\hat{x}_{t}^{-}} \quad M_{t}\left(\hat{x}_{t}^{-}\right)=\left.\frac{\partial h\left(x_{t}\right)}{\partial v_{t}}\right|_{x_{t}=\hat{x}_{t}^{-}}
$$

The numerical integration of ordinary differential equations (ODE) is used for the simulative propagation of Eq. (A.4), covariance matrix time update Eq. (A.7) and Jacobian matrix evaluation Eq. (A.6). Since variablestep solvers might be difficult to use for hard real-time applications requiring a deterministic processing time, this study is based on a fixed-step solver. A fixed step solver also tends to give more accurate calculation of finitedifference gradients since numerical errors are more systematic. Any of the fixed-step solvers can simulate the model to any desired level of accuracy, given a small enough step size. Unfortunately, it is generally difficult to decide a priori which combination of solver and step size will yield acceptable results for the continuous states in the shortest processing time. Determining the best solver for a particular model generally requires experimentation. In this study, the Matlab routine ode2 is used to propagate Eq. (A.4) and Eq. (A.7), which is the fixed step explicit Heun's method (Runge-Kutta method) (Ascher and Petzold, 1998)

$$
\begin{aligned}
\tilde{x}_{i+1} & =x_{i}+h f_{c}\left(x_{i}, u_{i}\right) \\
x_{i+1} & =x_{i}+\frac{h}{2}\left(f_{c}\left(x_{i}, u_{i}\right)+f_{c}\left(\tilde{x}_{i}, u_{i}\right)\right)
\end{aligned}
$$


where $i$ represents the numerical step index and $h$ is the numerical step size. It can be seen as an extension of the Euler method into a two-stage second-order Runge-Kutta method. Heun's method is a predictor-corrector method with the forward Euler's method, Eq. (A.12), as predictor and the trapezoidal method, (A.13), as corrector (Butcher, 2003). This method was chosen after some experimentation with a set of solvers. The precision of the numerical solution of a given solver as a function of the numerical step size $h$ is evaluated during the run of the EKF. In our case, the solver which gives the best EKF performance (least estimation errors) with other EKF settings unchanged, is chosen. The estimation error is however not the only criterion for how to choose the ODE solver. With regards to a real-time application the computational complexity and processing time need to be considered. The chosen Heun's method is a reasonable compromise between the computational time and solution precision with the given step size $h$. The Jacobian is approximated via the function's forward finite-difference to evaluate Eq. (A.6),

$$
\left.\frac{\partial f_{c}(x)}{\partial x}\right|_{x=\hat{x}} \approx\left[\frac{f_{c}\left(x+d_{1} \epsilon\right)-f_{c}(x)}{\epsilon}, \ldots, \frac{f_{c}\left(x+d_{n_{x}} \epsilon\right)-f_{c}(x)}{\epsilon}\right]_{x=\hat{x}}
$$

where $d_{i}$ is a unit vector in a direction of $x_{i}, i=1 \ldots n_{x}$ and $\epsilon$ represents a small perturbation.

Implementation of the EKF on a production ECU (Electronic Control Unit) poses some additional challenges, and some tradeoff between computational complexity and numerical accuracy. Computations of the Jacobian should either exploit the polynomial structure of the maps, or structural zeros in the Jacobian if finite differences are used. Robust and efficient numerical implementation of the EKF like Bierman's algorithm (Bierman, 1977) or the squre-root/array algorithm (Kailath et al., 2000) must be considered.

\section{Appendix B. Experimental and simulation setup}

Appendix B.1. Tested engine data and open loop performance of observer

The driving cycle US FTP75 is performed with a BMW 4-cylinder EU4 common rail direct injection (CRDI), production passenger car, turbocharged Diesel engine to record the data of the intake manifold pressure $p_{i}$, exhaust manifold pressure $p_{x}$, turbocharger speed $n_{t c}$ and the intake mass flow MAF sensor data. While the sensors have fast dynamics and therefore not modeled 
dynamically in our case study, such dynamic models in terms of simple lowpass filters could easily be added to the dynamic estimation model when the sensor dynamic is significant (Guardiola et al., 2013). The sampling interval ${ }^{1}$ for all the measurements is $\mathrm{T}=0.1[s]$. The engine is operated under standard laboratory conditions.

In the open loop mode the observer is not using any measured information to compute the estimated states except the model inputs (where $n_{e}$ is a measurement). In the open loop mode, the states are purely numerically simulated through the engine model from the given initial conditions of states and the given inputs. The inputs for the engine and the model are the percentages of open positions of vanes $X_{v g t}$ and EGR valve $X_{\text {egr }}$, fuel injection $W_{f}$ and the load which is reflected by the engine speed. The open loop observer gives biased estimates as previously documented in Polóni et al. (2012), where the biases are more significant on intake manifold pressure and turbocharger speed state and less evident on exhaust manifold pressure state.

\section{Appendix B.2. Numerical setup of computation}

Good tuning of the EKF depends on precise information about the stochastic properties of noises, which are theoretically assumed to be sequentially uncorrelated Gaussian distributions with zero means. The numerical values can be estimated by the user's careful assumption about the magnitude of measurement and process noises of a given state or a bias of a discrete (sampled) time sequence. After some manual tuning, the numerical values listed in Table B.2 are found to give the acceptable estimation and correction method performance for the engine used for the experiments.

The measurement noise covariance matrix is defined as

$$
R_{t}=\operatorname{diag}\left[\sigma_{y_{1}}^{2}, \sigma_{y_{2}}^{2}, \sigma_{y_{3}}^{2}\right]
$$

The process noise spectral density matrix is

$$
Q=\operatorname{diag}\left[\Phi_{1}, \Phi_{2}, \Phi_{3}, \Phi_{4}, \Phi_{5}, \Phi_{6}, \Phi_{7}, \Phi_{8}\right]
$$

where the diagonal noise spectral densities are defined and computed as $\Phi_{i}=(1 / T) \sigma_{i}^{2}$. The $\Phi_{1}$ spectral density depends on the intake manifold

\footnotetext{
${ }^{1}$ Note that the sampling interval $T$ differs from the integration step interval $h$
} 


\begin{tabular}{|c|c|}
\hline Sampling interval $T$ & $0.1[s]$ \\
\hline Integration step $h$ & $0.01[s]$ \\
\hline Finite difference perturbation $\epsilon$ & $10^{-8}$ \\
\hline Singular value threshold $\delta_{S V D}$ & $10^{-2} \max (D)$ \\
\hline Initial intake pressure $x_{1,0}$ & $9.8 * 10^{4}[\mathrm{kPa}]$ \\
\hline Initial intake mass $x_{2,0}$ & $0.01269[\mathrm{~kg}]$ \\
\hline Initial exhaust pressure $x_{3,0}$ & $9.8 * 10^{4}[k P a]$ \\
\hline Initial exhaust mass $x_{4,0}$ & $0.01269[\mathrm{~kg}]$ \\
\hline Initial turbocharger speed $x_{5,0}$ & $3.0 * 10^{4}[\mathrm{rpm}]$ \\
\hline Initial intake pressure derivative bias $w_{i, 0}$ & $0\left[k P a . s^{-1}\right]$ \\
\hline Initial exhaust pressure derivative bias $w_{x, 0}$ & $0\left[k P a . s^{-1}\right]$ \\
\hline Initial turbocharger speed derivative bias $w_{t c, 0}$ & $0\left[\right.$ rpm.s $\left.^{-1}\right]$ \\
\hline Initial intake pressure $p_{i}$ variance $\Sigma_{1,0}$ & $\left(0.3 * 10^{4}\right)^{2}\left[k P a^{2}\right]$ \\
\hline Initial intake mass $m_{i}$ variance $\Sigma_{2,0}$ & $\left(0.1 * 10^{-2}\right)^{2}\left[k g^{2}\right]$ \\
\hline Initial exhaust pressure $p_{x}$ variance $\Sigma_{3,0}$ & $\left(2 * 10^{3}\right)^{2}\left[k P a^{2}\right]$ \\
\hline Initial exhaust mass $m_{x}$ variance $\Sigma_{4,0}$ & $\left(1 * 10^{-4}\right)^{2}\left[\mathrm{~kg}^{2}\right]$ \\
\hline Initial turbocharger speed $n_{t c}$ variance $\Sigma_{5,0}$ & $\left(11 * 10^{3}\right)^{2}\left[\mathrm{rpm}^{2}\right]$ \\
\hline Initial intake pressure derivative bias $w_{i}$ variance $\Sigma_{6,0}$ & $\left(10^{5}\right)^{2}\left[\mathrm{kPa}^{2} \mathrm{~s}^{-2}\right]$ \\
\hline Initial exhaust pressure derivative bias $w_{x}$ variance $\Sigma_{7,0}$ & $\left(10^{5}\right)^{2}\left[k P a^{2} s^{-2}\right]$ \\
\hline $\begin{array}{l}\text { Initial turbocharger speed derivative bias } w_{t c} \text { variance } \Sigma_{8,0} \\
\text { Measurement noise: }\end{array}$ & $\left(10^{5}\right)^{2}\left[r p m^{2} s^{-2}\right]$ \\
\hline Standard deviation of the intake pressure $\sigma_{y_{1}}$ & $0.02 * 10^{5}[\mathrm{kPa}]$ \\
\hline Standard deviation of the exhaust pressure $\sigma_{y_{2}}$ & $0.08 * 10^{5}[k P a]$ \\
\hline $\begin{array}{l}\text { Standard deviation of the turbocharger speed } \sigma_{y_{3}} \\
\text { Process noise: }\end{array}$ & $0.1 * 10^{4}[\mathrm{rpm}]$ \\
\hline Standard deviation of the intake pressure $\sigma_{1}$ & $0.06 * 10^{5}[\mathrm{kPa}]$ \\
\hline Standard deviation of the intake mass $\sigma_{2}$ & $0.5 * 10^{-4}[\mathrm{~kg}]$ \\
\hline Standard deviation of the exhaust pressure $\sigma_{3}$ & $0.08 * 10^{5}[\mathrm{kPa}]$ \\
\hline Standard deviation of the intake mass $\sigma_{4}$ & $0.5 * 10^{-4}[\mathrm{~kg}]$ \\
\hline Standard deviation of the turbocharger speed $\sigma_{5}$ & $1.5 * 10^{4}[\mathrm{rpm}]$ \\
\hline Standard deviation of the intake pressure derivative bias $\sigma_{6}$ & $10^{4}\left[\mathrm{kPa}_{\mathrm{P}} \mathrm{s}^{-1}\right]$ \\
\hline Standard deviation of the exhaust pressure derivative bias $\sigma_{7}$ & $10^{4}\left[\mathrm{kPa}_{\mathrm{P}}^{-1}\right]$ \\
\hline Standard deviation of the turbocharger speed derivative bias $\sigma_{8}$ & $10^{4}\left[\mathrm{rpm}_{\mathrm{s}}^{-1}\right]$ \\
\hline
\end{tabular}

Table B.2: Settings of numerical computation 
pressure process noise variance $\sigma_{1}^{2}$ and sampling time, and is computed as $\Phi_{1}=10\left[s^{-1}\right]\left(0.06 * 10^{5}[k P a]\right)^{2}\left[k P a^{2} . s^{-2} . H z^{-1}\right]$, where the other spectral densities are similarly computed. The initial state vector estimate is set to $\hat{x}_{0}^{+}=\left[x_{1,0}, x_{2,0}, x_{3,0}, x_{4,0}, x_{5,0}, w_{i, 0}, w_{x, 0}, w_{t c, 0}\right]^{T}$. The initial covariance matrix of the initial state vector estimate error is

$$
P_{0}^{+}=\operatorname{diag}\left[\Sigma_{1,0}, \Sigma_{2,0}, \Sigma_{3,0}, \Sigma_{4,0}, \Sigma_{5,0}, \Sigma_{6,0}, \Sigma_{7,0}, \Sigma_{8,0}\right]
$$

where the diagonal elements are in accordance to Eq. (A.3) computed as $\Sigma_{i}=\left(x_{i, 0}-E\left[x_{i, 0}\right]\right)^{2}$ and set by the initial conditions.

\section{References}

Ascher, U. M., Petzold, L. R., 1998. Computer Methods for Ordinary Differential Equations and Differential-Algebraic Equations. SIAM.

Bierman, G. J., 1977. Factorization methods for discrete sequential estimation. Academic Press.

Brown, R. G., Hwang, P. Y. C., 2012. Introduction to Random Signals and Applied Kalman Filtering, 4th Edition. Wiley.

Butcher, J., 2003. Numerical Methods for Ordinary Differential Equations. John Wiley\&Sons, Ltd.

Desantes, J., Galindo, J., Guardiola, C., Dolz, V., 2010. Air mass flow estimation in turbocharged diesel engines from in-cylinder pressure measurement. Experimental Thermal and Fluid Science 34 (1), 37 - 47.

Diop, S., Moraal, P., Kolmanovsky, I., Van Nieuwstadt, M., 1999. Intake oxygen concentration estimation for DI diesel engines. In: Control Applications, 1999. Proceedings of the 1999 IEEE International Conference on. Vol. 1. pp. $852-857$ vol. 1.

Eriksson, L., Wahlström, J., Klein, M., 2010. Physical modeling of turbocharged engines and parameter identification. In: del Re, L., Allgöwer, F., Glielmo, L., Guardiola, C., Kolmanovsky, I. (Eds.), Automotive Model Predictive Control. Vol. 402 of Lecture Notes in Control and Information Sciences. Springer Berlin / Heidelberg, pp. 53-71. 
Gelb, A., Joseph F. Kasper, J., Raymond A. Nash, J., Price, C. F., Arthur A. Sutherland, J., 2001. Applied optimal estimation. The. M.I.T. Press.

Golub, G. H., Van Loan, C. F., 1996. Matrix computations, 3rd Edition. The Johns Hopkins University Press.

Guardiola, C., Pla, B., Blanco-Rodriguez, D., Mazer, A., Hayat, O., 2013. A bias correction method for fast fuel-to-air ratio estimation in diesel engines. Proceedings of the Institution of Mechanical Engineers, Part D: Journal of Automobile Engineering.

URL http://pid.sagepub.com/content/early/2013/03/28/ 0954407012473415. abstract

Heywood, J. B., 1988. Internal Combustion Engine Fundamentals. McGrawHill.

Höckerdal, E., Frisk, E., Eriksson, L., 2009. Observer design and model augmentation for bias compensation with a truck engine application. Control Engineering Practice 17 (3), $408-417$.

Höckerdal, E., Frisk, E., Eriksson, L., 2011. EKF-based adaptation of lookup tables with an air mass-flow sensor application. Control Engineering Practice 19 (5), $442-453$.

Jung, M., 2003. Mean-value modelling and robust control of the airpath of a turbocharged diesel engine. Ph.D. thesis, Sidney Sussex College, University of Cambridge.

Kailath, T., Sayed, A. H., Hassibi, B., 2000. Linear Estimation. Prentice Hall.

Kang, J.-M., Haskara, I., Chang, C.-F., Wang, Y.-Y., 2011. Adaptive intake oxygen estimation in a diesel engine. Patent Application, US 2011/0184632 A1.

Kolmanovsky, I., Moraal, P., van Nieuwstadt, M., Stefanopoulou, A., 1998. Issues in modelling and control of intake flow in variable geometry turbocharged engines. In: 18th IFIP Conference of Systems Modelling and Optimization. 
Kolmanovsky, I., Sivergina, I., Sun, J., 2006. Simultaneous input and parameter estimation with input observers and set-membership parameter bounding: theory and an automotive application. International Journal of Adaptive Control and Signal Processing 20 (5), 225-246.

Kolmanovsky, I. V., Jankovic, M. J., Van Nieuwstadt, M. J., Moraal, P. E., 2000. Method of estimating mass airflow in turbocharged engines having exhaust gas recirculation. Patent, US 6035639.

Polóni, T., Rohal-Ilkiv, B., Alberer, D., del Re, L., Johansen, T. A., 2012. Comparison of sensor configurations for mass flow estimation of turbocharged diesel engines. In: Alberer, D., Hjalmarsson, H., del Re, L. (Eds.), Identification for Automotive Systems. Vol. 418 of Lecture Notes in Control and Information Sciences. Springer, pp. 303-326.

Stefanopoulou, A. G., Storset, O. F., Smith, R., 2004. Pressure and temperature-based adaptive observer of air charge for turbocharged diesel engines. International Journal of Robust and Nonlinear Control 14 (6), $543-560$.

Storset, O. F., Stefanopoulou, A. G., Smith, R., 2004. Adaptive air charge estimation for turbocharged diesel engines without exhaust gas recirculation. Journal of Dynamic Systems, Measurement, and Control 126 (3), 633-643.

Stotsky, A., Kolmanovsky, I., 2002. Application of input estimation techniques to charge estimation and control in automotive engines. Control Engineering Practice 10 (12), 1371 - 1383.

van Nieuwstadt, M., Kolmanovsky, I., Moraal, P., Stefanopoulou, A., Jankovic, M., jun 2000. EGR-VGT control schemes: experimental comparison for a high-speed diesel engine. Control Systems Magazine, IEEE $20(3), 63-79$.

Wahlström, J., Eriksson, L., 2011. Modelling diesel engines with a variablegeometry turbocharger and exhaust gas recirculation by optimization of model parameters for capturing non-linear system dynamics. Proceedings of the Institution of Mechanical Engineers, Part D: Journal of Automobile Engineering 225 (7), 960-986.

URL http://pid. sagepub.com/content/225/7/960. abstract 
Wang, J., 2008. Air fraction estimation for multiple combustion mode diesel engines with dual-loop EGR systems. Control Engineering Practice 16 (12), $1479-1486$. 